\title{
Arterial Stiffness and Arterial Aging
}

\author{
Leticia Gomez-Sanchez ${ }^{1,2 *}$ and Marta Gomez-Sanchez ${ }^{1}$ \\ ${ }^{1}$ Primary Care Research Unit, the Alamedilla Health Center, Spain \\ ${ }^{2}$ Biomedical Research Institute of Salamanca (IBSAL), Spain
}

Received: February 27, 2018; Published: March 09, 2018

*Corresponding author: Leticia Gomez-Sanchez, Primary Care Research Unit, Alamedilla Health Care Center, Castilla and León, Health Service (SACyL), 37003 Salamanca, Spain, Tel: 34923 290900-53550; Email: leticiagmzsnchz@gmail.com

\section{Introduction}

The process of population ageing that is occurring in developed societies represents a major challenge for the health system. Cardiovascular diseases (CVD) are the most common cause of morbidity and mortality in the world, representing half of the deaths in people over 65 years old. Among the CVD, systemic arterial hypertension is related to more than half of those outcomes [1]. The non-modifiable cause of vascular ageing in healthy adults is age, because it causes increased arterial stiffness and changes in chromosome replication with telomere shortening [2]. The comparison of biological age with chronological age allows us to establish if the pattern of vascular ageing is at an expected or accelerated rate, which means if early vascular ageing is present or not $[1,2]$. Arterial stiffness is mainly determined by sex, age, and blood pressure [1,2].

\section{Age}

Arterial stiffness increases with age even in healthy adults [3]. In young individuals, arteries tend to be more elastic; therefore, the reflected wave occurs later in the cardiac cycle, reaching the heart during diastole. In older individuals, however, the reflected wave occurs earlier, reaching the heart during systole [1]. It involves changes in vascular structure with thickening of the arterial wall, changes in vascular function due to increased stiffness, and decreasing wall compliance of large arteries, which is associated with endothelial dysfunction and pulse wave changes $[4,5]$. These changes are primarily mediated by nitric oxide. Arterial ageing is a multifactorial process associated with changes in structure and function of the large arteries [1-3]. These changes are related to age, and are accelerated in the presence of associated CVD, cardiovascular risk factors or cardio-unhealthy lifestyles. Some of these factors are controllable, and a greater understanding of the links existing between vascular ageing, lifestyle and CVD will help to find strategies focused on preventing or delaying this ageing $[6,7]$.

\section{Sex}

Markers of arterial stiffness differ between men and women. Dyslipidemia and glucose contribute to a modest increase in arterial stiffness only in women. Therefore, the arteries of women may be more vulnerable to cardiovascular risk factors than those of men [8].

\section{Blood Pressure}

Arterial stiffness, measured by pulse wave velocity, is substantially increased in hypertension even in a way independently of blood pressure levels. Increased arterial stiffness has a major effect on pulse pressure (the difference between systolic and diastolic blood pressure), wave reflections, kidney function, and above all, cardiovascular risk and arterial ageing. This increased cardiovascular risk is particularly deleterious in patients with hypertension and/or diabetes mellitus type 2, who are at risk of both renal and cardiovascular events [9]. The Arterial Stiffness has been raised as an independent rate of risk for CVD, and it shows the dissociation between chronological age and biological age of the large arteries. Several prospective studies carried in general population as in subjects with cardiovascular risk factors have concluded that the arterial stiffness increased the morbimortality by CVD and general mortality. Four met analysis sump up the results of the main studies [10-13].

The Aortic Pulse Wave Velocity has been used in two of these studies to measure the Arterial Stiffness, including occidental subjects. The first review made by Vlachopoulous etal. [12], included 17 longitudinal studies, which have tracked 15.877 subjects with and without RCVF associated. The conclusion was that an increased of one $\mathrm{m} / \mathrm{s}$ of the aortic wave velocity raised the CVR in $14 \%$ for cardiovascular diseases and in 15\% for cardiovascular and general causes of mortality, after adjusts the results by age, sex and CVRF. The second one, made by Ben-Sholmo et al. [10], included the results of 16 longitudinal studies, which tracked 17.635 subjects with and without associated RCVF. It has been published that for each increase in one standard deviation of log of the aortic pulse wave velocity, the OR for coronary disease is 1.23 (IC 95\% 1.111.35); for the cerebral vascular stroke was 1.28 (IC 95\% 1.19-1.42) and for the cardiovascular events was 1,30 (IC 95\% 1, 18-1,43). The other two reviews have used as a measure of the arterial stiffness the brachial-ankle pulse wave velocity, including oriental subjects. 
The one carried out by Vlachopoulos et al. [13], included the results of 15 longitudinal studies, tracking 8.159 subjects. In this review, it was concluded that an increased of one $\mathrm{m} / \mathrm{s}$ of the Brachial Ankle Pulse Wave Velocity raised in 12\%, 13\% and 6\% the risk of cardiovascular events, the cardiovascular mortality and the mortality by all causes, respectively, after adjustment by age, sex and RCV factors. The Brachial-Ankle Pulse Wave Velocity predictive capacity was better in the youngest patients. Finally, the recent review made by Ohkuma $\mathrm{T}$ et al. [11], included 14.673 Japanese subjects of 14 prospective studies, without previous events of CVD. This one concluded that each increase of $1 \mathrm{DE}$ of the BrachialAnkle Pulse Wave Velocity was associated with a raise in the OR of 1.19 (IC 95\% 1.10-1.29) for suffering cardiovascular mortal events and non mortal after adjustment by classics factor of CVR. Arterial stiffness can be assessed with different clinical tools. The tools more frequently used are arterial aplannation tonometry (it requires previous training by the person who carries it out) and oscilometry (easy to use it) [14].

It has been suggested that the oscilometry is the best technique for the evaluation of VOP in the clinical daily use, because of its precision, easy-use, reliability and robustness. The techniques that use a measure based on a sleeve on one arm or wrist only are encouraging. The arterial stiffness non-invasive measures most used are the aortic pulse wave velocity, the Brachial-Ankle Pulse Wave Velocity and the Cardio-ankle vascular index (CAVI). The aortic pulse wave velocity measured by tonometry or Doppler is known as the gold standard for the assessment of arterial stiffness [14]. The Aortic Pulse Wave Velocity, measured by applanation tonometry, is widely used in clinical and observational studies, but its clinical use has not been instituted due to its high price and the technical difficulties and previous training required. New devices that measure the arterial stiffness by tonometry make easier their use in the clinical practice [15].

The Brachial-Ankle Pulse Wave Velocity is measured automatically by using a pressure cuff on each of the four limbs, and because of its easy-use and simplicity, it could be used in the daily practice [16]. To end with, the CAVI measured by VASERA device is an accuracy repeatable and reproducible method. It is easy to use and it doesn't cause injurious to the patients. The CAVI is not influenced by the value of the blood pressure in the moment of doing the measure [17]. Nevertheless, the huge number of different parameters used to determinate the arterial stiffness and also the different techniques of measuring, has blocked the clinical impact. On the other hand, each of the parameter mentioned above shows a different characteristic of the arteriosclerosis process, which means the various functional and/or morphologic changes of the vessel Wall.

Therefore, measuring simultaneous different parameters of the vascular function and structure could improve the global assessment of the vascular ageing in each patient. Another important consideration to bear on mind is if the measure has showed independent predictive value for cardiovascular events.
This fact has been proved with the aortic pulse wave and with the Brachial-Ankle Pulse Wave Velocity $[10,12]$ but not with the CAVI $[11,13]$. To sum up, actually the arterial stiffness could be accurate, reliable and easy assessed. It also could be useful as a biomarker to predict the value of the arterial ageing. But prospective studies are needed to compare the different ways to measure Arterial Stiffness, and so the different devices, and to analyze which is the most adequate measure and dispositive to use both of them in the daily clinical practice.

\section{References}

1. Mikael LR, Paiva AMG, Gomes MM, Sousa ALL, Jardim P, et al. (2017) Vascular Aging and Arterial Stiffness. Arq Bras Cardiol 109(3): 253-258.

2. Lee HY, Oh BH (2010) Aging and arterial stiffness. Circ J 74(11): 22572262.

3. Veerasamy M, Ford GA, Neely D, Bagnall A, MacGowan G, et al. (2014) Association of aging, arterial stiffness, and cardiovascular disease: a review. Cardiol Rev 22(5): 223-232.

4. Nilsson PM (2014) Hemodynamic Aging as the Consequence of Structural Changes Associated with Early Vascular Aging (EVA). Aging Dis 5(2): 109-113.

5. Oakley R, Tharakan B (2014) Vascular hyperpermeability and aging. Aging Dis 5(2): 114-125.

6. Artandi SE (2006) Telomeres, telomerase, and human disease. N Engl J Med 355(12): 1195-1197.

7. Maruyama Y (2012) Aging and arterial-cardiac interactions in the elderly. Int J Cardiol 155(1): 14-19.

8. Kim JY, Park JB, Kim DS, Kim KS, Jeong JW, et al. (2014) Gender Difference in Arterial Stiffness in a Multicenter Cross-Sectional Study: The Korean Arterial Aging Study (KAAS). Pulse (Basel) 2(1-4): 11-17.

9. Safar ME (2018) Arterial stiffness as a risk factor for clinical hypertension. Nat Rev Cardiol 15(2): 97-105.

10. Ben-Shlomo Y, Spears M, Boustred C, May M, Anderson SG, et al. (2014) Aortic pulse wave velocity improves cardiovascular event prediction: an individual participant meta-analysis of prospective observational data from 17, 635 subjects. J Am Coll Cardiol 63(7): 636-646.

11. Ohkuma T, Ninomiya T, Tomiyama H, Kario K, Hoshide S, et al. (2017) Brachial-Ankle Pulse Wave Velocity and the Risk Prediction of Cardiovascular Disease: An Individual Participant Data Meta-Analysis. Hypertension 69(6): 1045-1052.

12. Vlachopoulos C, Aznaouridis K, Stefanadis C (2010) Prediction of cardiovascular events and all-cause mortality with arterial stiffness: a systematic review and meta-analysis. J Am Coll Cardiol 55(13): 13181327.

13. Vlachopoulos C, Aznaouridis K, Terentes-Printzios D, Ioakeimidis N, Stefanadis C (2012) Prediction of cardiovascular events and all-cause mortality with brachial-ankle elasticity index: a systematic review and meta-analysis. Hypertension 60(2): 556-562.

14. Laurent S, Marais L, Boutouyrie P (2016) The Noninvasive Assessment of Vascular Aging. Can J Cardiol 32(5): 669-679.

15. Zhang Y, Yin P, Xu Z, Xie Y, Wang C, et al. (2017) Non-Invasive Assessment of Early Atherosclerosis Based on New Arterial Stiffness Indices Measured with an Upper-Arm Oscillometric Device. Tohoku J Exp Med 241(4): 263-270.

16. Ninomiya T, Kojima I, Doi Y, Fukuhara M, Hirakawa Y, et al. (2013) Brachial-ankle pulse wave velocity predicts the development of 
cardiovascular disease in a general Japanese population: the Hisayama Study. J Hypertens 31(3): 477-483.

\section{(c) (i)}

Submission Link: https://biomedres.us/submit-manuscript.php
This work is licensed under Creative Commons Attribution 4.0 License
17. Shirai K, Hiruta N, Song M, Kurosu T, Suzuki J, et al. (2011) Cardio-ankle vascular index (CAVI) as a novel indicator of arterial stiffness: theory, evidence and perspectives. J Atheroscler Thromb 18(11): 924-938.

$\begin{array}{ll}\text { BIOMEDICAL } & \text { Assets of Publishing with us } \\ \text { RESEARCHES } & \text { - Global archiving of articles } \\ \text { - Immediate, unrestricted online access } & \text { - Rigorous Peer Review Process } \\ & \text { - Authors Retain Copyrights } \\ \end{array}$

